\title{
How Nitrogen Doping Affects Hydrogen Spillover on Carbon-Supported Pd Nanoparticles: New Insights from DFT
}

\author{
Lisa Warczinski* and Christof Hättig \\ Ruhr-University Bochum, Universitätsstraße 150, 44780 Bochum, Germany \\ E-mail: lisa.warczinski@rub.de
}

\section{Hückel calculations}

For the Hückel calculations we used two sets of parameters to account for the two types of N atoms:

- pyridinic nitrogens, N1: $\beta_{N 1-C}=1.00 \beta_{C-C}$ and $\alpha_{N 1}=\alpha+0.50 \beta$

- graphitic nitrogens, $\mathrm{N} 2: \beta_{N 2-C}=0.89 \beta_{C-C}$ and $\alpha_{N 2}=\alpha+1.37 \beta$

where $\alpha$ is the atomic orbital energy of $\mathrm{C}$ atoms and $\beta$ are the reduced resonance integrals between two $\mathrm{C}$ atoms. 


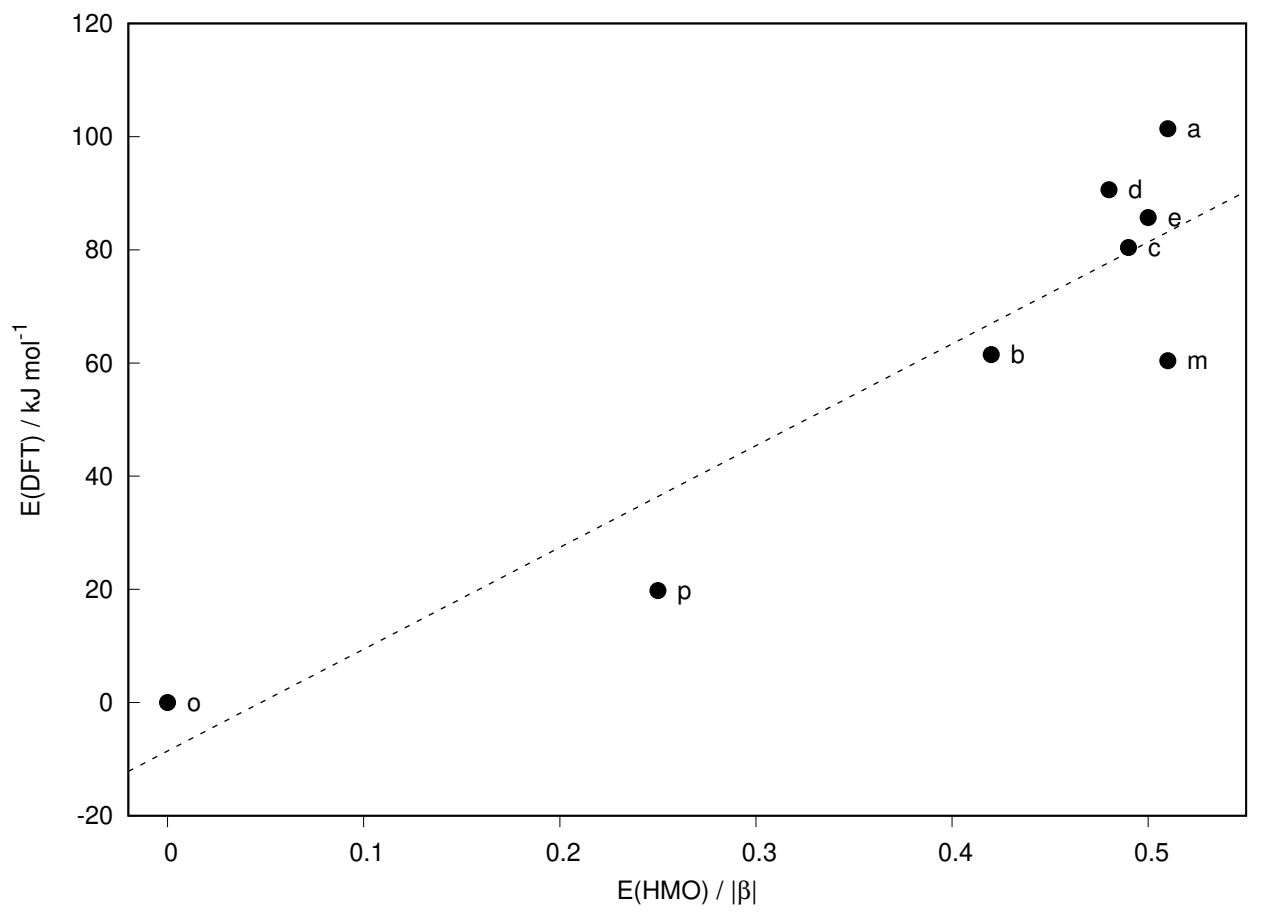

Figure S1: Correlation between the DFT and Hückel binding energies for spillover hydrogen for the different positions with respect to graphitic $N$ (cmp. Table 1 of the main text). The dashed line shows the result of a least square fit of $\Delta E(\mathrm{DFT})=c+\beta \Delta E(\mathrm{HMO})$ that resulted in $c=-8.6 \mathrm{~kJ} \mathrm{~mol}^{-1}$ and $\beta=-179.8 \mathrm{~kJ} \mathrm{~mol}^{-1}$. 
a

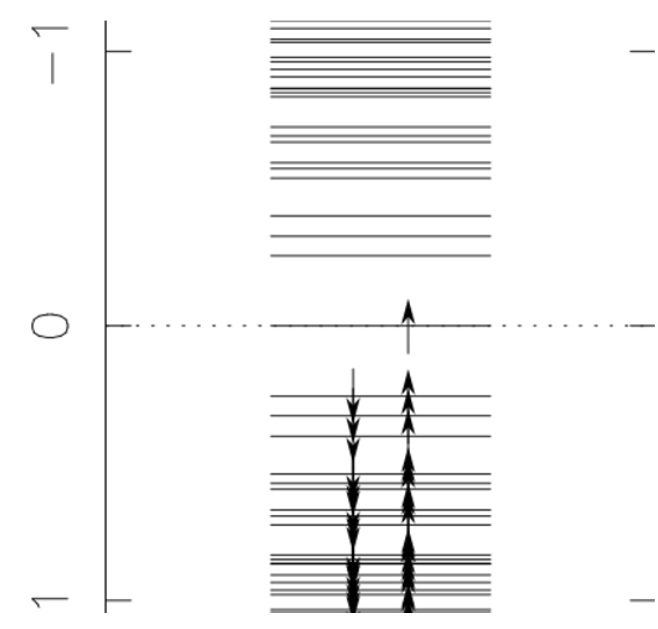

b

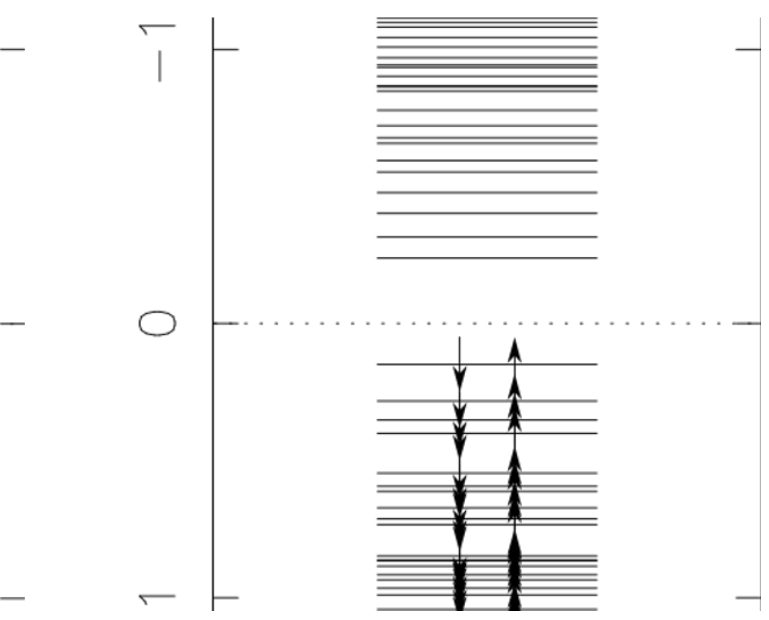

Figure S2: Sketch of Hückel orbitals: (a) one spillover hydrogen connected to one carbon atom of the graphene sheet; (b) one spillover hydrogen connected to one carbon atom directly next to a graphitic $\mathrm{N}$ atom. 


\section{Stability with respect to DFT functional and basis set}

Table S1: Comparison of activation energies for the hydrogen spillover from $\mathrm{Pd}_{21}$ onto different support materials on the basis of TPSS/def2-SVP geometries.

\begin{tabular}{l|c|c|c}
\hline model system & $\begin{array}{c}\text { TPSS/ } \\
\text { def2-SVP }\end{array}$ & $\begin{array}{c}\text { PBE/ } \\
\text { def2-SVP }\end{array}$ & $\begin{array}{c}\text { TPSS/ } \\
\text { def2-TZVP }\end{array}$ \\
\hline $\mathrm{Pd}_{21}$ on graphene support; triangle site & 130.8 & 130.7 & 127.5 \\
$\mathrm{Pd}_{21}$ on graphitic N support; bridge site & 46.8 & 45.5 & 46.3
\end{tabular}

Table S2: Comparison of activation energies for spillover hydrogen diffusion on different support materials on the basis of TPSS/def2-SVP geometries.

\begin{tabular}{c|c|c|c}
\hline pathway & $\begin{array}{c}\text { TPSS/ } \\
\text { def2-SVP }\end{array}$ & $\begin{array}{c}\text { PBE/ } \\
\text { def2-SVP }\end{array}$ & $\begin{array}{c}\text { TPSS/ } \\
\text { def2-TZVP }\end{array}$ \\
\hline a & 105.0 & 94.3 & 106.6 \\
b & 121.5 & 114.5 & 120.1
\end{tabular}

Table S3: Comparison of activation energies for the change from the chemisorbed to the physisorbed state on the basis of TPSS/def2-SVP geometries.

\begin{tabular}{l|c|c|c}
\hline model system & $\begin{array}{c}\text { TPSS/ } \\
\text { def2-SVP }\end{array}$ & $\begin{array}{c}\text { PBE/ } \\
\text { def2-SVP }\end{array}$ & $\begin{array}{c}\text { TPSS/ } \\
\text { def2-TZVP }\end{array}$ \\
\hline $\begin{array}{l}\text { pure graphene support } \\
\text { graphitic N support }\end{array}$ & 68.5 & 76.6 & 66.5 \\
107.6 & 114.0 & 106.5
\end{tabular}




\section{Electron density differences}
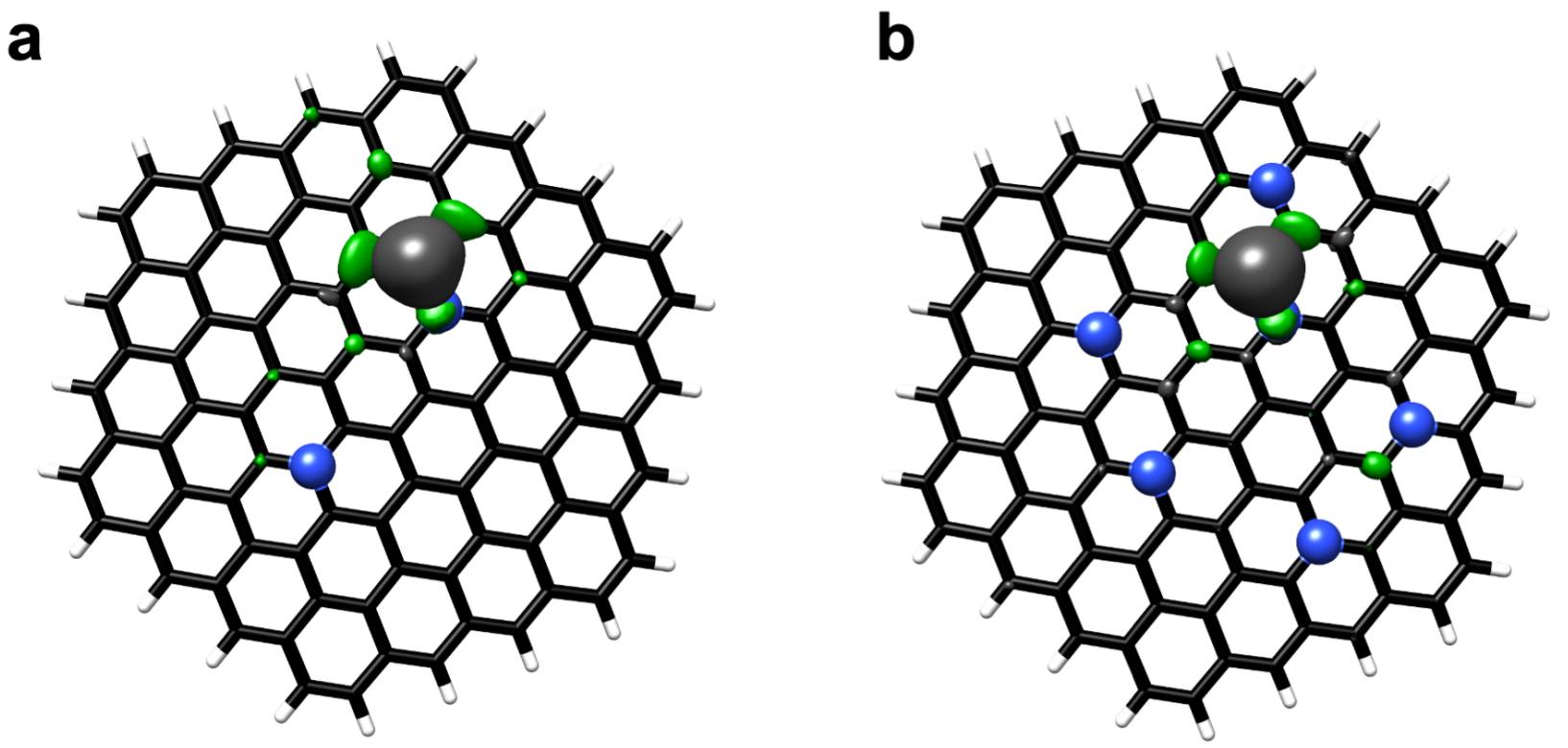

Figure S3: Electron density difference plots for spillover hydrogen on the graphitic N support material: (a) two graphitic N atoms; (b) six graphitic N atoms. Density gain is colored in green; density loss is colored in grey. (isosurface values: \pm 0.0025 ) 


\section{Spin density}

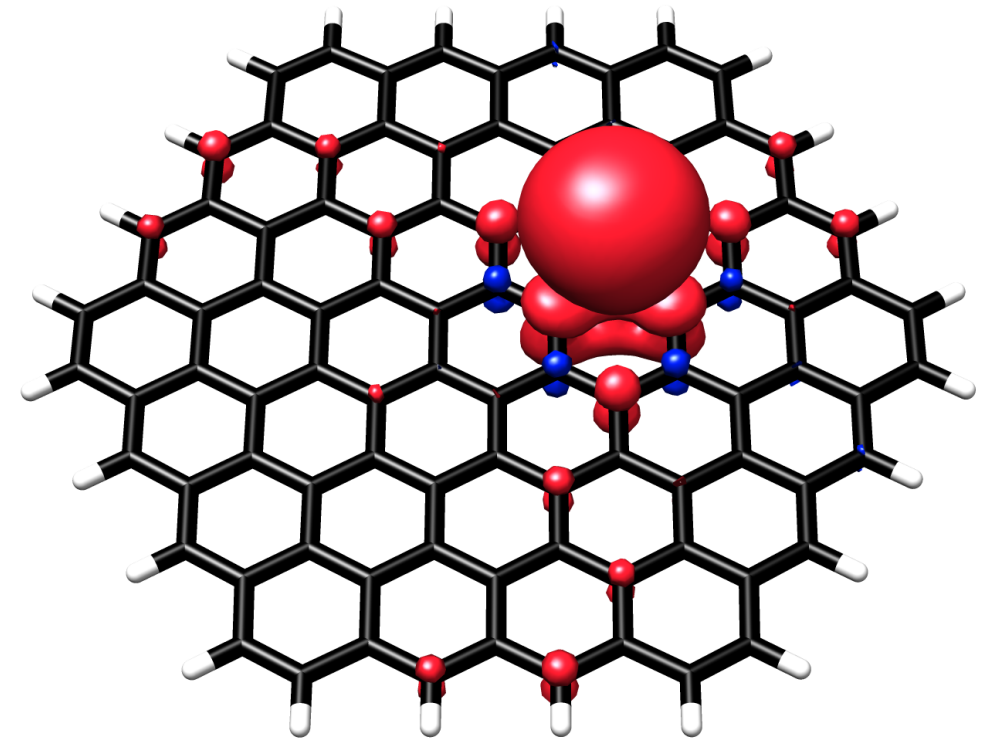

Figure S4: Plot of the spin density for spillover hydrogen in the physisorption minimum. (isosurface values: \pm 0.0002 ) 


\section{Additional reaction pathways}

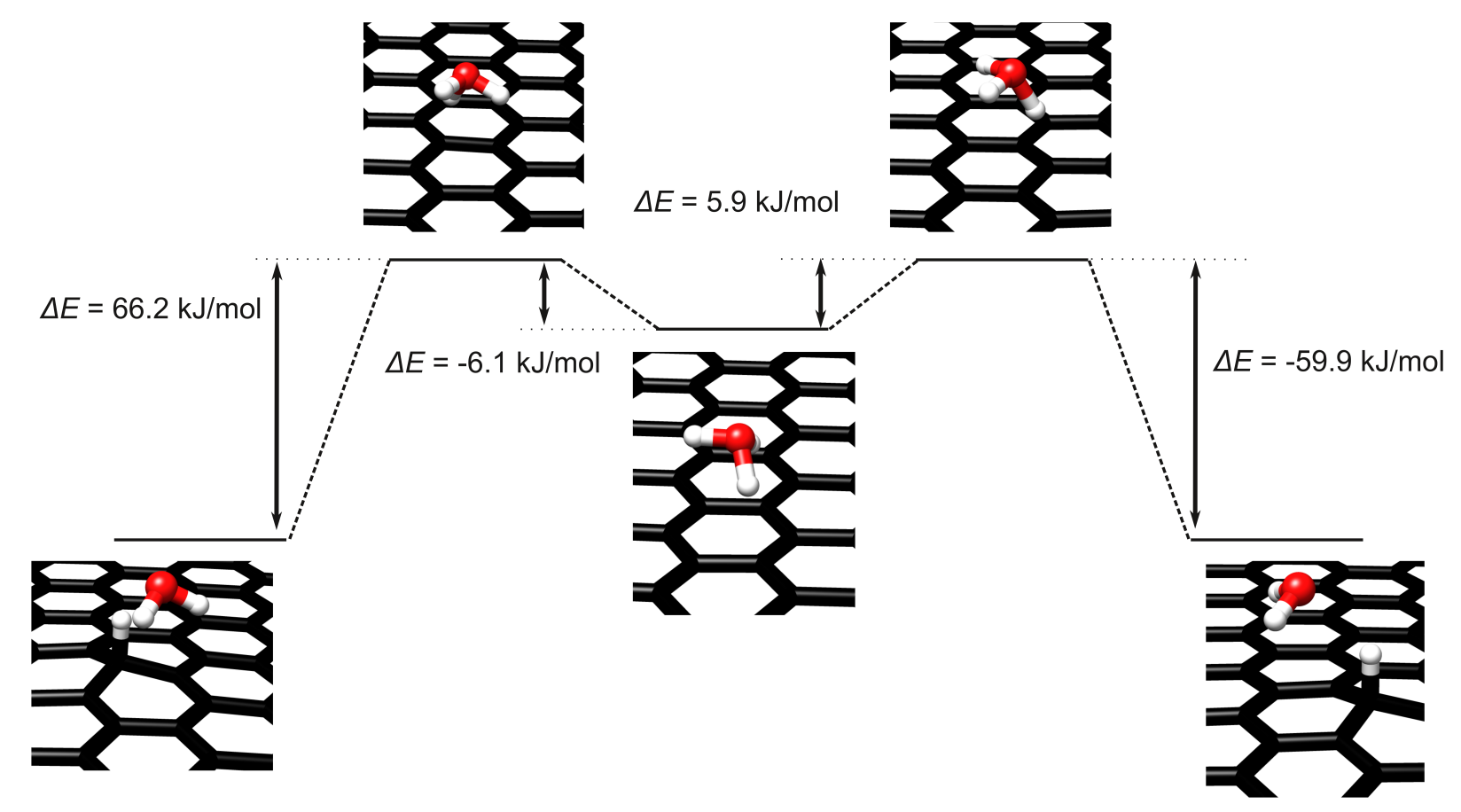

Figure S5: Reaction pathway for spillover hydrogen diffusion via the Grotthus mechanism.

Table S4: Energies and free activation enthalpy differences for spillover hydrogen diffusion via the Grotthus mechanism.

\begin{tabular}{l|c|c}
\hline & $\Delta E / \mathrm{kJ} \mathrm{mol}^{-1}$ & $\Delta G / \mathrm{kJ} \mathrm{mol}^{-1}$ \\
\hline React. - TS 1 & +66.2 & +77.7 \\
TS 1 - Min. & -6.1 & -1.4 \\
Min. - TS 2 & +5.9 & +0.4 \\
TS 2 - Prod. & -59.9 & -73.4
\end{tabular}




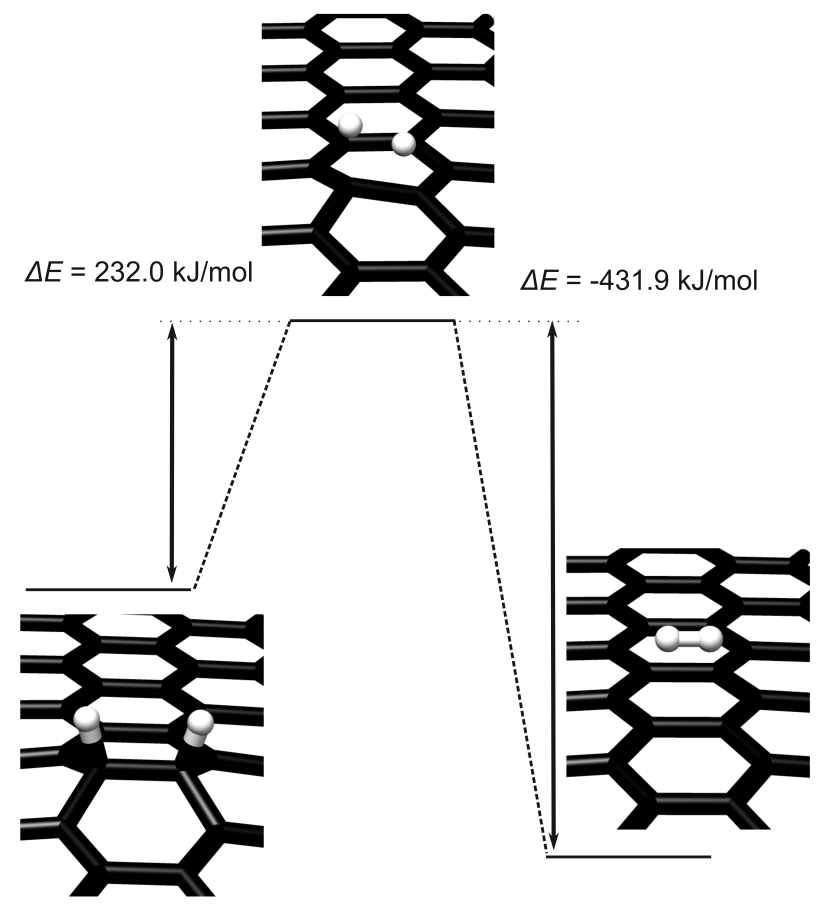

Figure S6: Hydrogen desorption pathway on the pure graphene support.

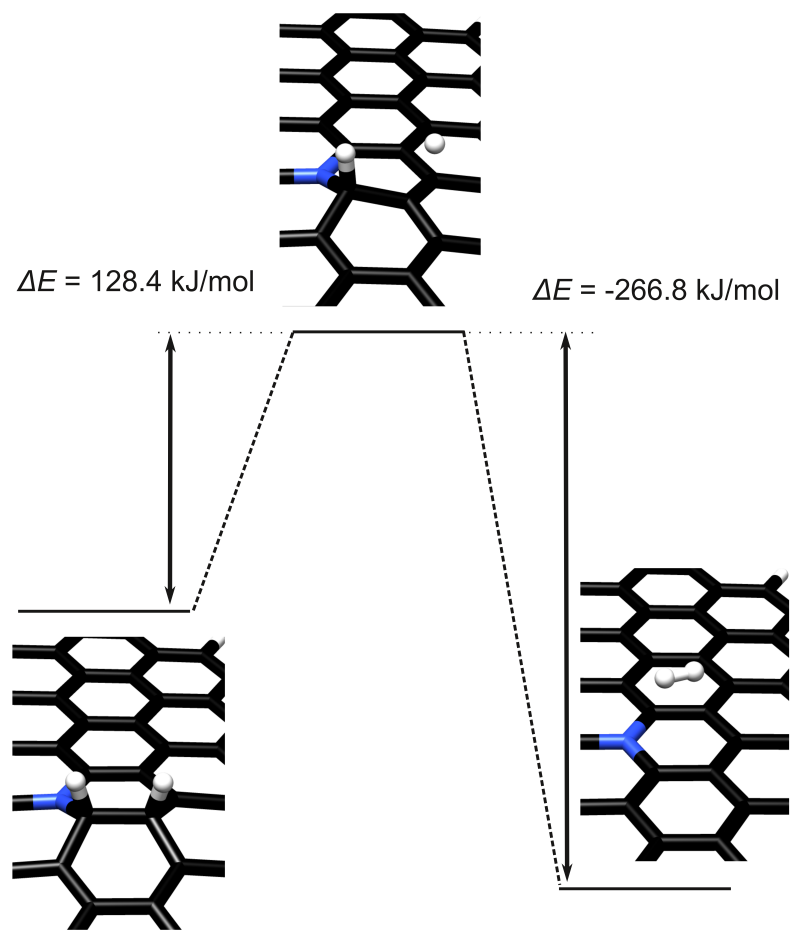

Figure S7: Hydrogen desorption pathway next to a graphitic N atom. 


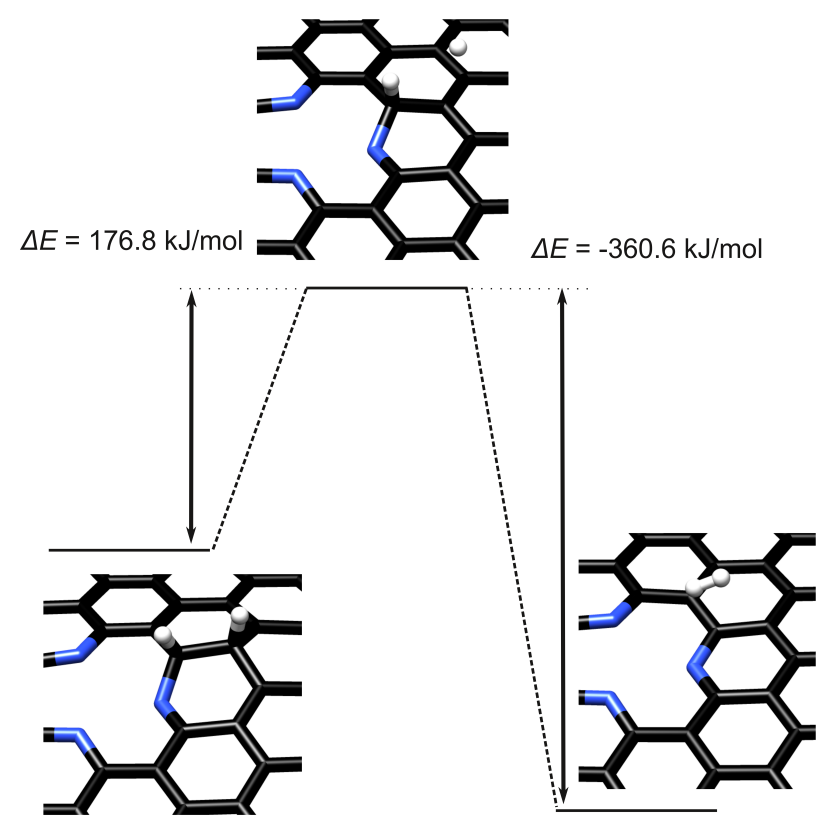

Figure S8: Hydrogen desorption pathway next to a pyridinic $\mathrm{N}$ atom.

\section{Different energy contributions}

Table S5: Different energy contributions to the binding energy of hydrogen on carbon in the physisorption minimum. The dispersion contribution has been taken as the D3 correction that is included in the total DFT-D3 binding energies (at the DFT-D3 geometries).

\begin{tabular}{l|c}
\hline & $E / \mathrm{kJ} \mathrm{mol}^{-1}$ \\
\hline total binding energy & -10.2 \\
ZPVE correction & +4.5 \\
Dispersion correction & -9.0
\end{tabular}

